\title{
Aortic sinus flow stasis likely in valve-in-valve transcatheter aortic valve implantation
}

\author{
Hoda Hatoum, BS, ${ }^{\text {a }}$ Brandon L. Moore, PhD, ${ }^{b}$ Pablo Maureira, MD, PhD, ${ }^{c}$ Jennifer Dollery, RN, ${ }^{\mathrm{d}}$ \\ Juan A. Crestanello, MD, ${ }^{\mathrm{d}}$ and Lakshmi Prasad Dasi, $\mathrm{PhD}^{\mathrm{a}, \mathrm{d}}$
}

\begin{abstract}
Objective: Valve-in-valve procedures using transcatheter aortic valves are increasingly performed to treat degenerated bioprosthetic surgical aortic valves because they are less invasive than redo aortic valve replacement. The objective of this study is to quantify the changes in aortic sinus blood flow dynamics before and after a valve-in-valve procedure to gain insight into mechanisms for clinical and subclinical thrombosis of leaflets.
\end{abstract}

Methods: A detailed description of the sinus hemodynamics for valve-in-valve implantation was performed in vitro. A Medtronic Hancock II (Medtronic Inc, Minneapolis, Minn) porcine bioprosthesis was modeled as a surgical aortic valve, and Medtronic CoreValve and Edwards Sapien (Edwards Lifesciences, Irvine, Calif) valves were used as the transcatheter aortic valves. High-resolution particle image velocimetry was used to compare the flow patterns from these 2 valves within both the left coronary and noncoronary sinuses in vitro.

Results: Velocity and vorticity within the surgical valve sinuses reached peak values of $0.7 \mathrm{~m} / \mathrm{s}$ and $1000 \mathrm{~s}^{-1}$, with a $70 \%$ decrease in peak fluid shear stress near the aortic side of the leaflet in the noncoronary sinus. With the introduction of transcatheter aortic valves, peak velocity and vorticity were reduced to approximately $0.4 \mathrm{~m} / \mathrm{s}$ and $550 \mathrm{~s}^{-1}$ and $0.58 \mathrm{~m} / \mathrm{s}$ and $653 \mathrm{~s}^{-1}$ without coronary flow and $0.60 \mathrm{~m} / \mathrm{s}$ and $631 \mathrm{~s}^{-1}$ and $0.81 \mathrm{~m} / \mathrm{s}$ and $669 \mathrm{~s}^{-1}$ with coronary flow for the CoreValve and Sapien valve-in-valve implantations, respectively. Peak shear stress was approximately $38 \%$ higher along the aortic side of the coronary versus noncoronary transcatheter aortic valve leaflet.

Conclusions: Decreased flow and shear stress in valve-in-valve procedures indicate a higher risk of leaflet thrombosis secondary to flow stasis, perhaps more so in the noncoronary sinus. (J Thorac Cardiovasc Surg 2017;154:32-43)

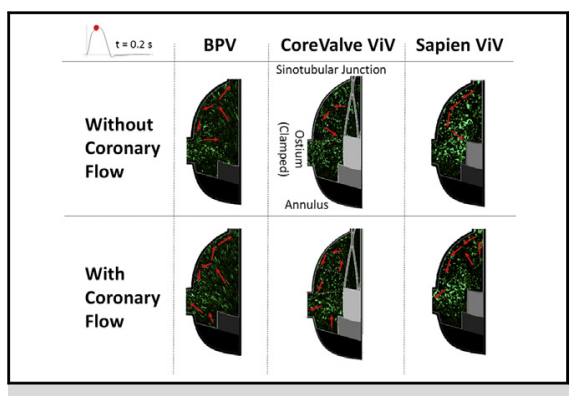

Greatly reduced flow seen in the sinuses with a ViV implantation.

\section{Central Message}

Implantation of TAVs in failed surgical bioprosthetic valve causes potential for flow stasis leading to possible leaflet thrombosis.

\section{Perspective}

ViV configurations can significantly lead to decreased sinus flow with and without coronary flow. Since stasis is often associated with thrombus formation, care must be taken in valve positioning to maximize sinus flow.

See Editorial Commentary page 44.
Elderly patients with a degenerated surgical bioprosthetic aortic valve are being increasingly considered for a valvein-valve $(\mathrm{ViV})$ procedure using a transcatheter aortic valve

From the Departments of ${ }^{\mathrm{a}}$ Biomedical Engineering and ${ }^{\mathrm{d}}$ Surgery, The Ohio State University, Columbus, Ohio; ${ }^{\mathrm{b}}$ Department of Mechanical Engineering, Colorado State University, Fort Collins, Colo; and ${ }^{\mathrm{C}}$ Department of Cardiovascular Surgery, CHU de Nancy, Nancy, France.

This work was partially supported by the National Institutes of Health under Award Number R01HL119824 and the American Heart Association under Award 11SDG5170011. The content is solely the responsibility of the authors and does not necessarily represent the official views of the National Institutes of Health or American Heart Association.

Received for publication Nov 28, 2016; revisions received Feb 14, 2017; accepted for publication March 11, 2017; available ahead of print April 19, 2017.

Address for reprints: Lakshmi Prasad Dasi, PhD, Department of Biomedical Engineering, The Ohio State University, 473 W 12th Ave, Columbus, OH 43210 (E-mail: lakshmi.dasi@osumc.edu).

$0022-5223 / \$ 36.00$

Copyright $\odot 2017$ by The American Association for Thoracic Surgery

http://dx.doi.org/10.1016/j.jtcvs.2017.03.053
(TAV) because of its less-invasive nature compared with conventional redo aortic valve replacement surgery. ${ }^{1}$ However, recent studies have shown a common occurrence of thrombosis-related leaflet immobilization in $\mathrm{TAV}^{2-4}$ In our recent letter, we eluded to one physical mechanism supported by a meta-analysis of data focused on observed thrombosis on leaflets. ${ }^{5}$ Therefore, it is critical to further investigate the detailed hemodynamics of the ViV configuration to elucidate the underlying hemodynamic

Scanning this QR code will take you to supplemental figures and video for this article. 


\section{Abbreviations and Acronyms \\ $\mathrm{BPV}=$ bioprosthetic heart valve \\ $\mathrm{TAV}=$ transcatheter aortic valve \\ $\mathrm{ViV}=$ valve-in-valve}

mechanism and predict the risk of thrombosis in future $\mathrm{ViV}$ procedures.

TAVs constitute a relatively new and exciting technology in the field of valvular heart disease. Although TAV currently is approved only for patients with a high risk of surgical complications, such as the elderly and those with severe comorbidities, it is widely being adopted for $\mathrm{ViV}$ use as a viable alternative for redo aortic valve surgery in patients with a failing bioprosthetic valve. The challenges that the ViV paradigm faces stem from some complications associated mainly with the underlying principles of TAV technology itself. Chief among these complications is the difficulty of placement. This difficulty can lead to breaking off of tissue and numerous other detrimental effects, ${ }^{6}$ such as blockage of coronary arteries, ${ }^{7,8}$ paravalvular regurgitation, and unfavorable hemodynamic environments that leave the patient susceptible to stroke. ${ }^{9,10}$

In this study, we seek to provide a detailed and quantitative description of the sinus hemodynamics in the case of $\mathrm{ViV}$ implantation and characterize the effects of left coronary flow on the possibility of sinus flow stasis. Sinus flow governs leaflet washout, and its characteristics in a $\mathrm{ViV}$ configuration can pose additional likelihood of stasis compared with conventional TAV implantation because of the additional narrowing of the aortic annulus from the sewing ring and the presence of stent-posts that interact with the sinus blood flow. Evaluating the mechanistic effects of this arrangement, particularly from the standpoint of sinus hemodynamics, may give an insight into the significant risk of leaflet thrombosis and its subsequent role in elevating gradients. ${ }^{11-13}$

\section{MATERIALS AND METHODS}

Full details of our methodology have been published ${ }^{14,15}$ in the context of another study. Briefly, 2-dimensional particle image velocimetry experiments were conducted to visualize aortic sinus flow corresponding to an isolated bioprosthetic heart valve (BPV) configuration and subsequently $2 \mathrm{ViV}$ arrangements in vitro. Velocity fields were captured within the noncoronary and coronary sinuses. The hemodynamic performance of these configurations was assessed in a pulse duplicator setup under physiologic pressure $(120 / 80 \mathrm{~mm} \mathrm{Hg})$ and flow $(5 \mathrm{~L} / \mathrm{min}){ }^{11}$ Data acquisition over 10 cycles was performed for each valve setup.

\section{Valve Models}

A 23-mm Medtronic Hancock II T505 (Medtronic, Minneapolis, Minn) porcine bioprosthetic aortic valve was mounted inside a clear, acrylic sinus chamber machined to mimic the outer walls of the aorta (Figure E1), based on the study by Yap and colleagues. ${ }^{16}$ The Hancock valve (Medtronic) was used as a model for an isolated bioprosthetic implanted aortic valve and tests were run with and without a TAV inserted into this isolated valve configuration.

The TAVs used were a 26-mm Medtronic CoreValve (Medtronic Inc) and a 23-mm Edwards Sapien (Edwards Lifesciences, Irvine, Calif), which were chosen to match annular size with the isolated bioprosthetic valve. Their positions relative to the isolated bioprosthetic valve were determined on the basis of clinical findings, which suggest an ideal TAV leaflet location that extends just downstream of the BPV valve leaflets. ${ }^{17}$ The CoreValve was chosen to be placed subannularly with respect to the BPV annulus. This valve combination and TAV placement location also compare well with published recommendations for ViV implantation. ${ }^{18,19}$

\section{RESULTS}

Differences in flow patterns between the isolated bioprosthetic valve coronary and noncoronary sinuses have been analyzed and discussed in detail in our previous work. ${ }^{15}$

\section{Coronary Flow Profile}

Coronary flow waveforms are presented in Figure E2 for the isolated bioprosthetic configuration and the $\mathrm{ViV}$ configurations. As shown in Figure E2, coronary flow is slightly reduced during systole in the presence of the TAV. The diastolic portions of the flow waveforms were not significantly different.

\section{Qualitative Sinus Flow Visualization}

High-resolution particle image velocimetry was used to measure and analyze the hemodynamics for 6 sinus cases: (1) isolated BPV configuration noncoronary, (2) isolated BPV configuration coronary, (3) CoreValve ViV configuration noncoronary, (4) CoreValve ViV configuration coronary, (5) Sapien ViV configuration noncoronary, and (6) Sapien ViV configuration coronary. Qualitative "streak" images were created for each case by computing a sliding average and subtracting the sliding minimum over 10 frames at a time. Depictions of camera and laser orientation, as well as viewing the orientation of the results, are shown in Figure E3. Corresponding videos of these particle streaks are included for 1 complete cardiac cycle for isolated BPV noncoronary, isolated BPV coronary, CoreValve ViV noncoronary, CoreValve ViV coronary, Sapien ViV noncoronary, and Sapien ViV coronary configurations as shown in Video 1. Still-frame snapshots from these videos are included in Figure 1.

The results for the isolated bioprosthetic valve configuration control cases are shown for the noncoronary (Figure 1, $A$ ) and coronary (Figure 1,D) sinuses. During early systole, forward flow in each of these cases is significant and sinus flow velocity is high. A stagnation point near the downstream end of the sinus wall where flow is split is diverted toward the sinotubular junction or recirculated back into the sinus appears. This point is located slightly farther upstream in the coronary sinus and migrates downstream in both cases as systole progresses. At midsystole, a flow 


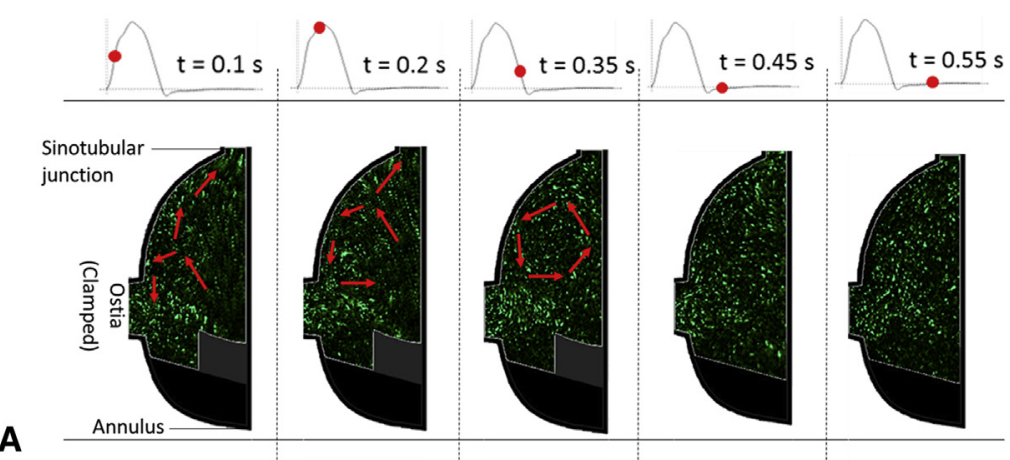

A

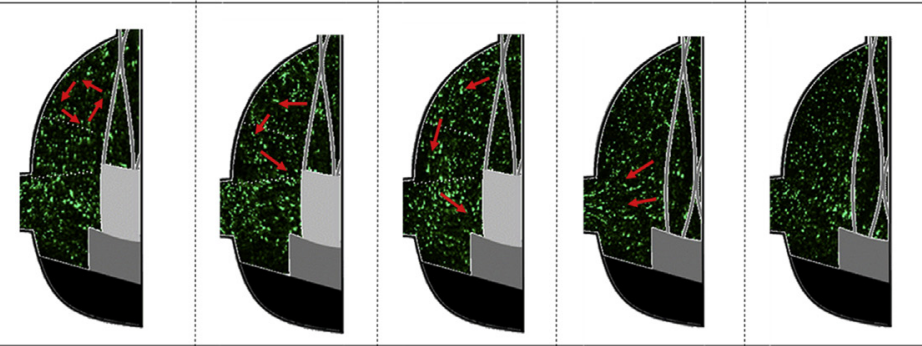

B

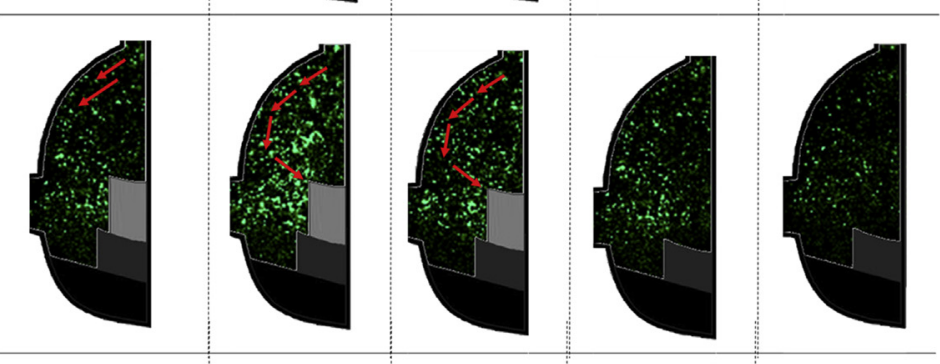

C

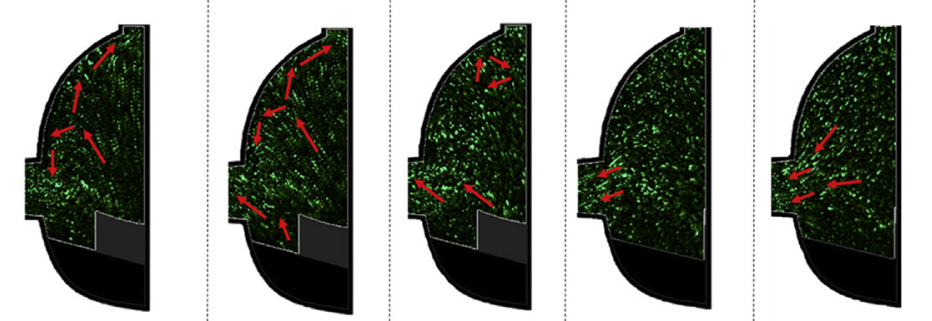

D

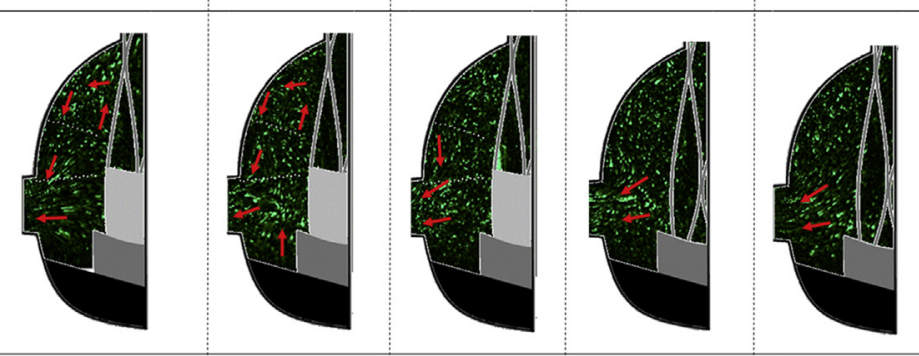

E

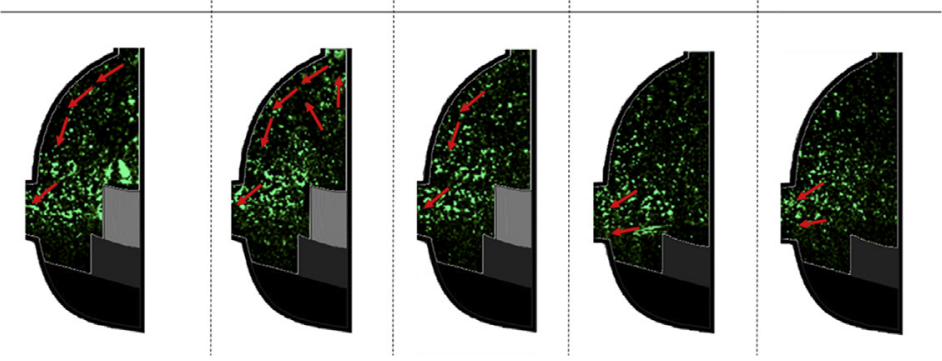

\section{$\mathbf{F}$}

FIGURE 1. Snapshots from streak plot videos generated by time bin averaging raw images of noncoronary sinuses for (A) BPV, (B) ViV with the CoreValve (Medtronic Inc, Minneapolis, Minn), and (C) ViV with the Sapien (Edwards Lifesciences, Irvine, Calif) and of coronary sinuses for (D) BPV, (E) ViV with the CoreValve, and (F) ViV with Sapien. Arrows are manually drawn (not computed vectors) to help depict trends in the video. 
from the back of the sinus takes place and then gets drawn into the coronary artery, which is absent in the noncoronary sinus case. Near the end of systole, the sinus vortex has grown; however, the flow velocity is significantly reduced. During diastole, sinus flow velocities are lower. There is a pattern of flow toward the base of the sinus at early diastole, but by mid-diastole the flow is relatively stagnant. In the coronary sinus, early and mid-diastole display particle motion that is mainly toward the ostium, with this pattern being stronger and more widespread throughout the sinus toward mid diastole.

Having a ViV setup greatly alters sinus hemodynamics (Figure 1, $B$ and $C$ ). There is little to no streamwise flow within the sinus during early systole; however, a small vortex is shed off the leaflet tip in the CoreValve ViV compared with a flow following the sinus wall contour and starting at the sinotubular junction in the Sapien ViV. This vortex grows in size and starts to fill more of the sinus as systole progresses. In the Sapien ViV, the flow starts to fill the sinus until the leaflet edge. However, the flow speed is lower for both ViV configurations for these portions of systole. Diastolic hemodynamics are similar with and without ViV.

Coronary flow in the ViV cases causes some changes in sinus hemodynamics from the noncoronary $\mathrm{ViV}$ case (Figure 1, E and $F$ ). During the early stages of systole in the CoreValve ViV, the sinus vortex covers more of the sinus. In the Sapien ViV, the flow tracks the sinus contour more obviously. However, flow does not recirculate completely but rather exits the sinus at the coronary ostium leading to no noticeable sinus vortex toward the end of systole. Likewise, both early and mid-diastolic hemodynamics show a dominant pattern of flow from all parts of the sinus toward the coronary ostium, with somewhat higher velocities during mid-diastole.

\section{Quantitative Flow Results}

The velocity vectors and vorticity contours are presented at select time-points of interest in Figures 2 and 3. In the BPV configuration (Figures 2, A, and 3,A), the presence of the sinus vortex is clear in the early stages of systole. The center of this vorticity is just downstream of the leaflet tip, in between the edge of the leaflet and the sinus wall. The velocity of the significant forward flow that enters the sinus reaches approximately $1.3 \mathrm{~m} / \mathrm{s}$ and the peak vorticity magnitude approximately $800 \mathrm{~s}^{-1}$. Most of this is diverted into the aorta by the curvature of the downstream sinus wall while a small portion is redirected back toward the base of the sinus. At midsystole, the sinus vortex is still clearly distinguished but has migrated slightly downstream. Vorticity magnitude is greatest at this time point, with a peak at approximately $1000 \mathrm{~s}^{-1}$ and vortex velocities on the order of $0.7 \mathrm{~m} / \mathrm{s}$. Late systole induces a reduction in the overall sinus velocity and therefore vorticity magnitude. However, a weakened sinus vortex is still present, which is the dominant sinus flow pattern because most streamwise flow no longer exists within the sinus. In the coronary sinus, the major velocity and vorticity magnitudes during diastole exist only near the ostium.

The most dramatic difference caused by the introduction of a TAV to the BPV (Figure 2, $B$ and $C$ ) is a reduction in overall velocity and vorticity magnitudes, which becomes noticeable during early systole. Also, the less noticeable sinus vortex is positioned more downstream in the TAV cases than in the BPV case. At midsystole, the sinus vortex size is relatively unchanged as it stretches from the sinotubular junction to the tip of the TAV leaflet. Vorticity magnitude also remains similar to that during early systole, with a peak of approximately $550 \mathrm{~s}^{-1}$ in the CoreValve ViV and $653 \mathrm{~s}^{-1}$ in the Sapien ViV. Velocity magnitude is reduced relative to the BPV case, with highest systolic values of approximately $0.4 \mathrm{~m} / \mathrm{s}$ and $0.58 \mathrm{~m} / \mathrm{s}$ in the CoreValve and Sapien ViV, respectively.

Velocity and vorticity were calculated for the coronary sinus for the ViV configurations (Figure 3, $B$ and $C$ ). In this case, the sinus vortex plays less of a role in the CoreValve ViV. For instance, at early systole, the highest velocity and vorticity magnitude occur as the result of flow entering the coronary ostium, with velocity magnitude in this region at approximately $0.3 \mathrm{~m} / \mathrm{s}$. In the Sapien ViV, the flow is directed by the coronary flow, so the motion at the sinus wall is more defined compared with the noncoronary case. At early systole, the highest velocity magnitude is approximately $0.45 \mathrm{~m} / \mathrm{s}$. Around midsystole, hemodynamics are not significantly changed; however, there is stronger recirculation around the downstream sinus wall and some capturing of the flow into the ostium is apparent. Once forward velocity has slowed, toward the end of systole, there is only a weak recirculating flow pattern present. Coronary flow increases by mid-diastole, reaching velocities of approximately $0.4 \mathrm{~m} / \mathrm{s}$ and $0.53 \mathrm{~m} / \mathrm{s}$ in the CoreValve and Sapien ViV, respectively.

\section{Shear Stress Distribution}

Shear stress was calculated at the given time points of interest for each valve case. Results are presented as contour maps in Figures 4 and 5 that give an indication on the order of magnitude of shear stresses in the sinus. The subregion near the leaflet is of particular interest because low leaflet wall shear stress may be linked to flow stasis.

BPV configuration sinus shear stress patterns are displayed in Figures 4, A, and 5, A. During early systole, most of the sinus is dominated by large magnitude negative (acting downward relative to respect to leaflet surface) shear stress, more than $1 \mathrm{~Pa}$ magnitude, in the downstream end of the sinus. However, there is also a significant region of strong positive shear adjacent to the leaflet free edge, which extends to the aortic side of the leaflet in the coronary cases. 


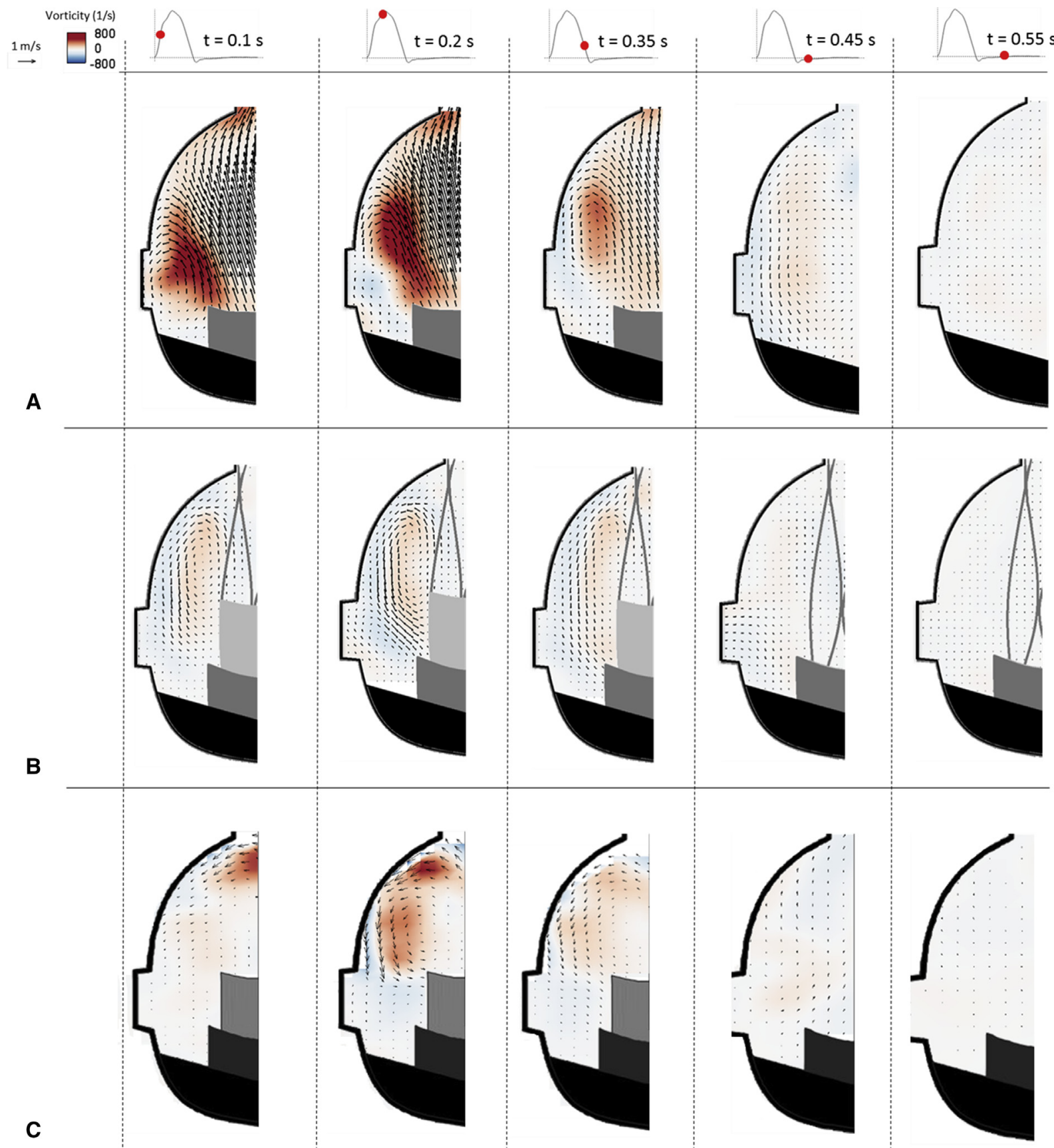

FIGURE 2. Velocity vectors and vorticity contours within the noncoronary sinus for (A) BPV, (B) ViV with CoreValve, and (C) ViV with Sapien at selected time points throughout the cardiac cycle.

During diastole, significant shear stress exists only near the edges of the ostium in the coronary sinus.

In the ViV configurations, sinus shear stress levels are generally reduced compared with the BPV cases (Figure 4, $B$ and $C$ ). During systole, in the CoreValve ViV 2 distinct shear regions are noticeable: one negative region along the aortic side of the TAV leaflet and one positive region along the sinus wall. Stress magnitude in these regions increases during midsystole and then decreases dramatically during late systole. In the Sapien ViV, the 2 distinct shear regions along the aortic side of the TAV and along the sinus wall are clear in early systole. Similar to the CoreValve ViV, stress magnitudes in these shear regions increase during midsystole and decrease during late systole. However, the pattern becomes more blended at peak systole and dissipates in late systole with a more dominating 


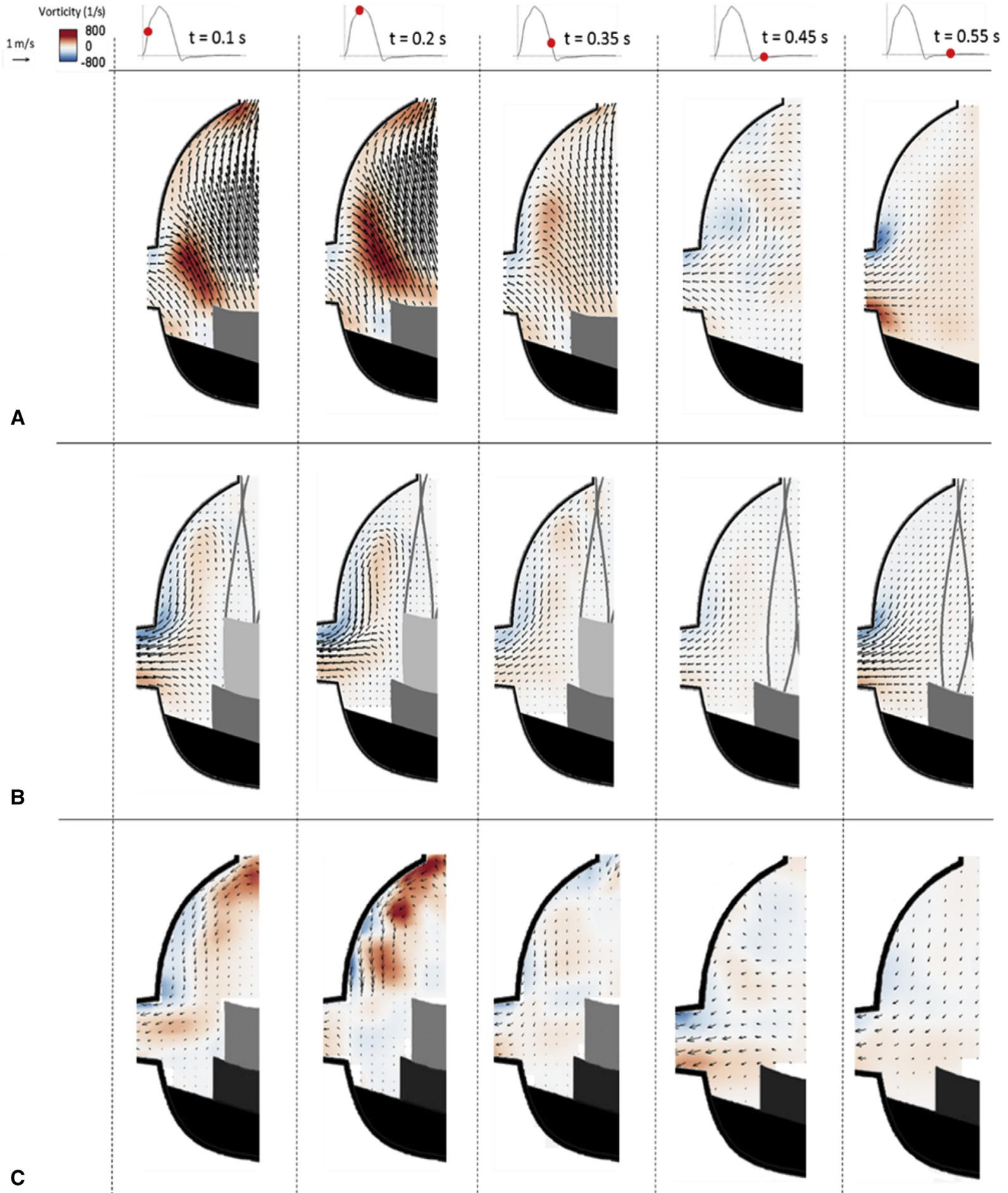

FIGURE 3. Velocity vectors and vorticity contours within the coronary sinus for (A) BPV, (B) ViV with CoreValve, and (C) ViV with Sapien at selected time points throughout the cardiac cycle.

negative region. There is little shear stress in the sinus throughout diastole for the ViV noncoronary cases.

Shear stresses for the ViV coronary sinus case are presented in Figure 5, $B$ and $C$. In the CoreValve ViV, there is higher magnitude shear stress near the leaflet tip and the ostium than in the noncoronary sinus (Figure 5, B). The same observation applies to the Sapien ViV near the ostium and inside the sinus compared with the noncoronary case (Figure 5,C), but this maximum shear stress region does not occur near the leaflet of the TAV. This observation 


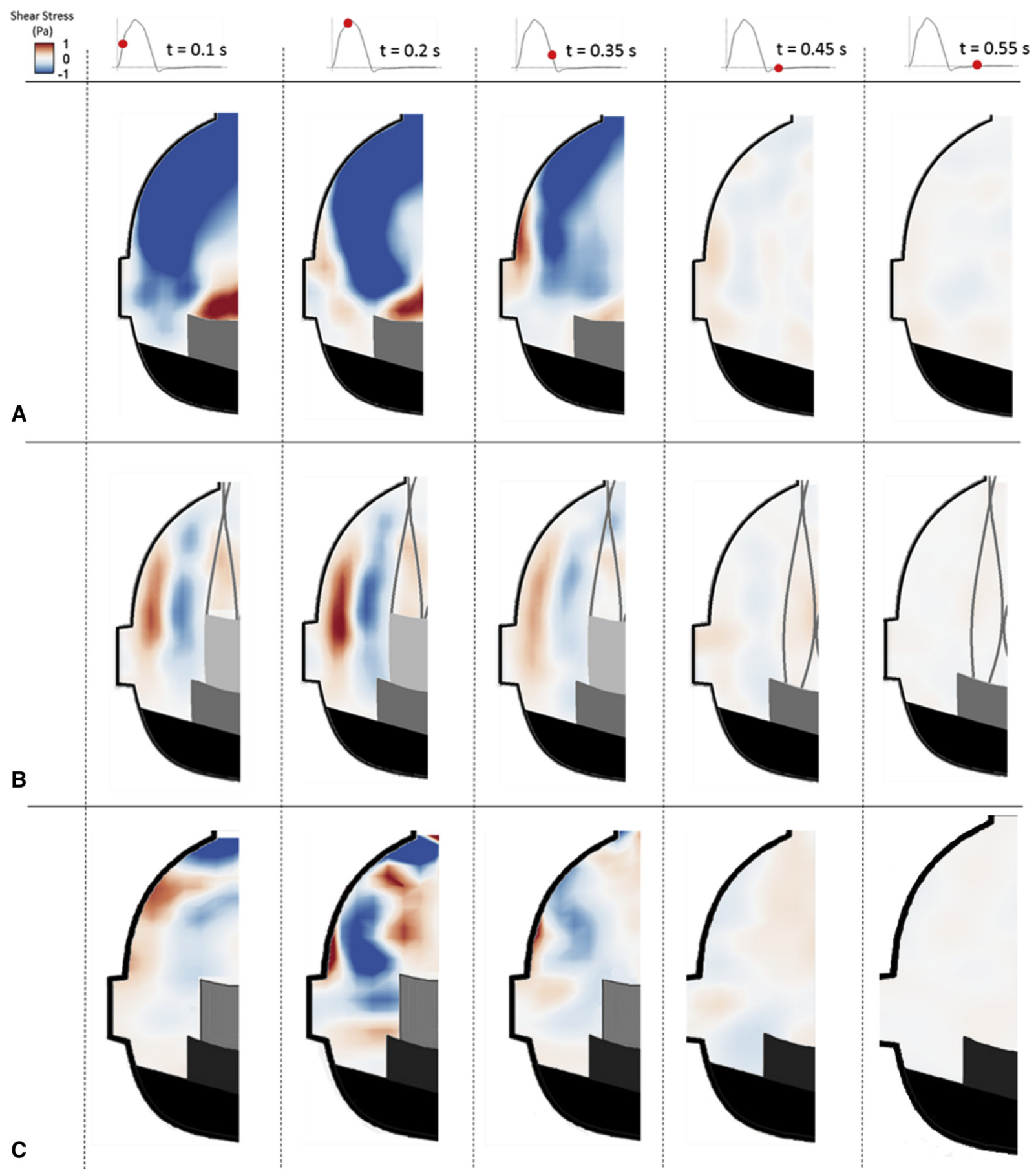

FIGURE 4. Shear stress contours within the noncoronary sinus of (A) BPV, (B) ViV with CoreValve, and (C) ViV with Sapien at selected time points throughout the cardiac cycle.

is more noticeable in the coronary case compared with the noncoronary case.

Figure $6, A$ and $B$, show the probability density function of flow shear stress magnitude in the subregion adjacent to the leaflets during systole and diastole, respectively. As is clearly evident in Figure 6, higher shear stresses $(>1.5 \mathrm{~Pa})$ occur in the BPV cases during systole alone. For the
CoreValve ViV cases, coronary flow appears to slightly augment the likelihood of even higher shear stresses $(>1.5 \mathrm{~Pa})$. For the ViV cases, shear stresses do not exceed approximately $1.4 \mathrm{~Pa}$ during systole. During diastole, the shear stress is less than $1.6 \mathrm{~Pa}$, with the noncoronary cases yielding a higher probability of shear stress exceeding $0.5 \mathrm{~Pa}$ but less than 1.6 Pa. For the Sapien ViV noncoronary 


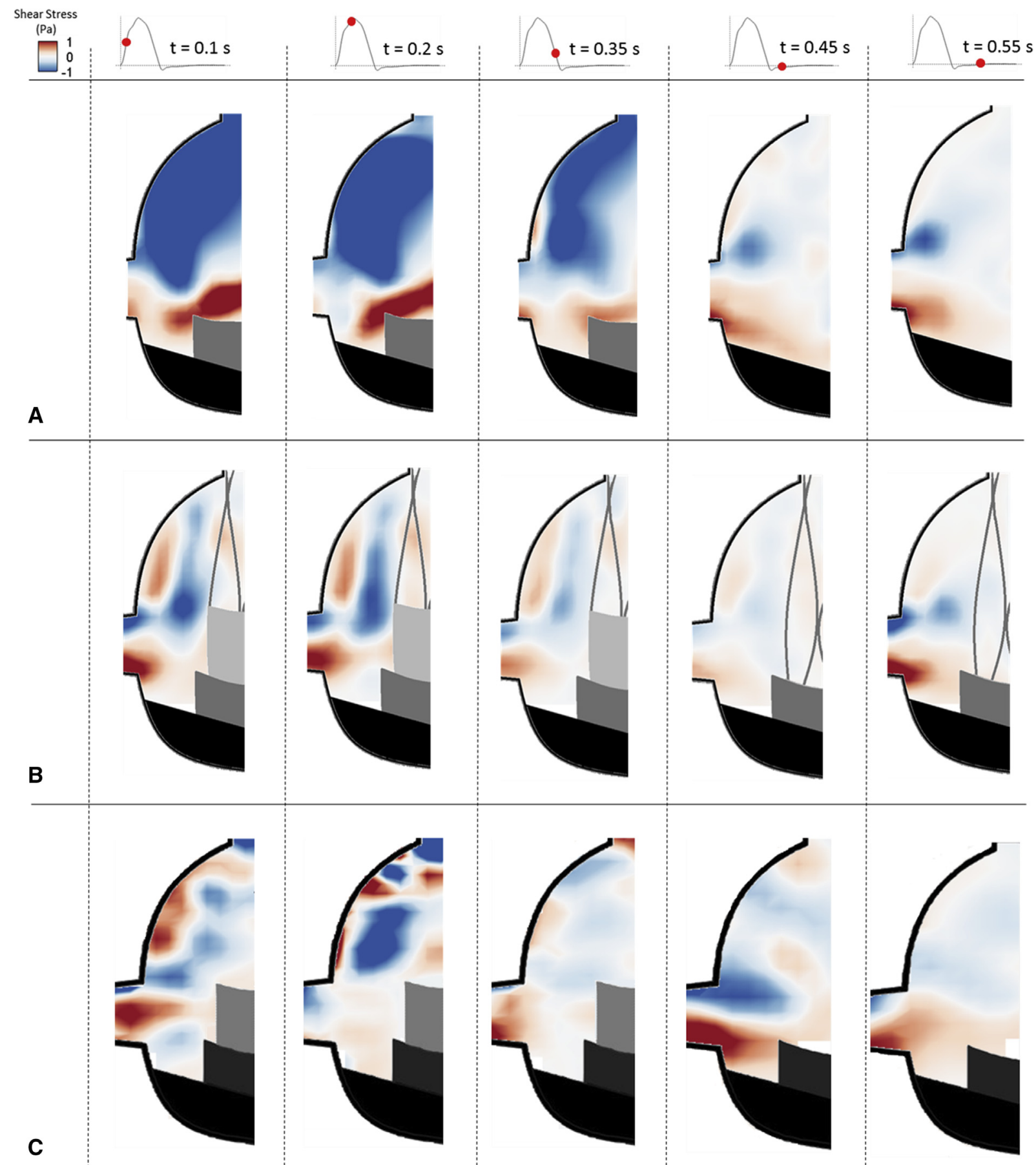

FIGURE 5. Shear stress contours within the coronary sinus of (A) BPV, (B) ViV with CoreValve, and (C) ViV with Sapien at selected time points throughout the cardiac cycle.

case during systole, higher shear stress magnitudes than $0.5 \mathrm{~Pa}$ likelihood decreases drastically near the leaflet region. However, shear stress reaches magnitudes up to 1.8 Pa compared with 1.1 Pa for the noncoronary CoreValve ViV. Coronary flow seems to have a significant impact on the shear stress probability distribution. High shear stress values have a high likelihood of occurrence near the leaflets before a gradual decrease occurs. During diastole, the probability of having high magnitudes of shear stress decreases with the coronary flow, yielding a higher probability of occurrence of higher magnitudes of shear stress near the leaflets. In systole and diastole, the Sapien ViV shows a drastic decrease in the likelihood of having shear stress ranging from 0.5 to $1.0 \mathrm{~Pa}$ and 0.2 to $0.5 \mathrm{~Pa}$ in systole and 

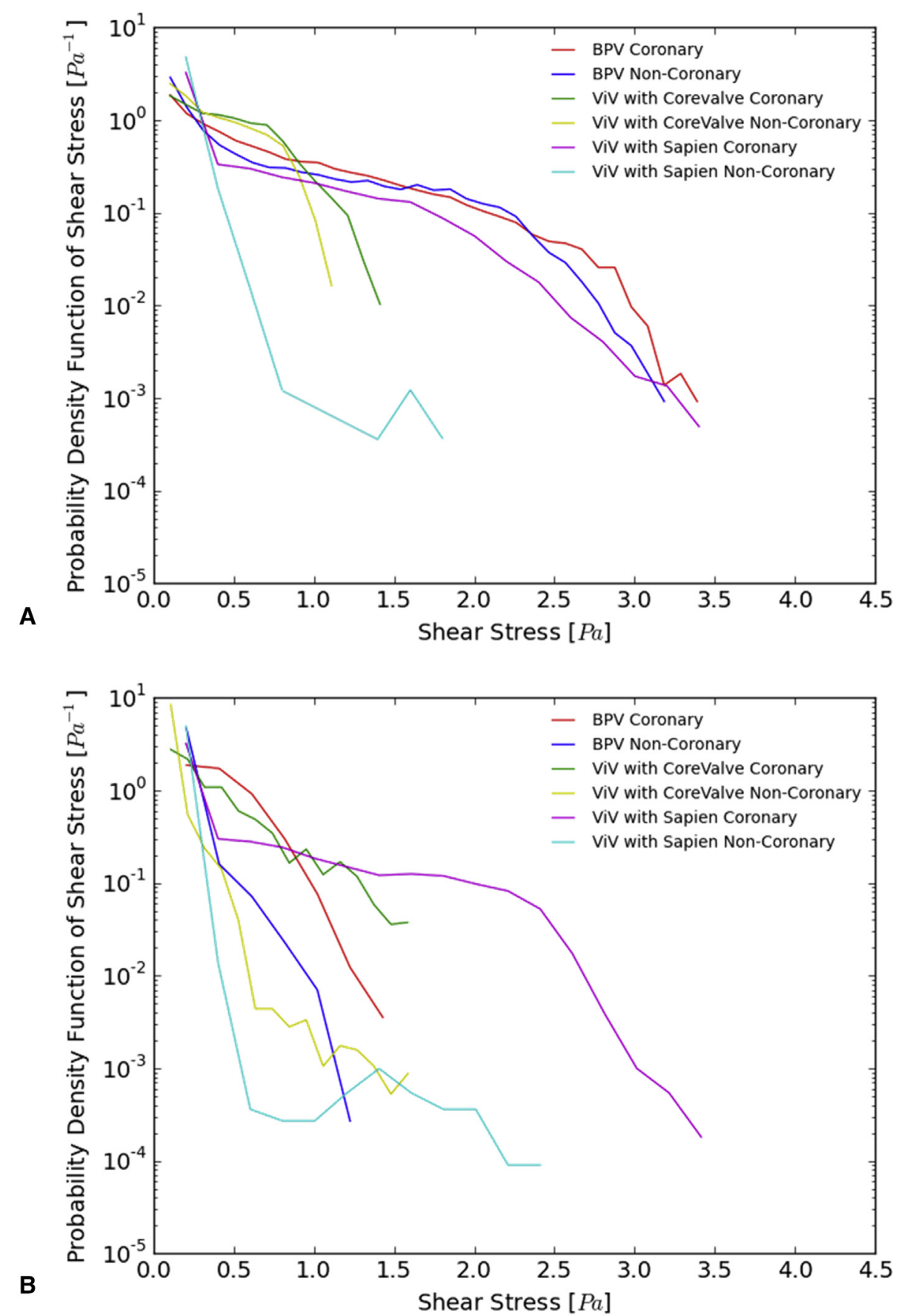

FIGURE 6. Probability density function in log scale of varying shear stress distribution values along a subregion near the isolated bioprosthetic and ViV configuration leaflets during (A) systole and (B) diastole. BPV, Bioprosthetic heart valve; $V i V$, valve-in-valve.

diastole, respectively, compared with patterns shown for the CoreValve ViV and the BPV in the noncoronary cases.

Table 1 encompasses leaflet washout velocity and mean shear stress magnitude in the region neighboring the leaflets in each valve case and shows the impact of coronary and noncoronary flow presence in addition to the differences between BPV and ViV. Also, mean values of pressure gradients that are in accordance with literature $^{19}$ are reported to be $5.01 \pm 0.0064 \mathrm{~mm} \mathrm{Hg}, 17.05 \pm 0.14 \mathrm{~mm}$ $\mathrm{Hg}$, and $17.67 \pm 0.27 \mathrm{~mm} \mathrm{Hg}$ for the BPV, CoreValve ViV, and Sapien ViV, respectively. Because ViV setups, especially with BPVs with sewing rings, compromise the annulus area and add more structures that alter the basic fluid dynamics (attachment and separation points, and shear layers), the pressure gradients are expected to be higher. The pressure gradient difference between the Sapien and 
TABLE 1. Mean shear stress magnitude and mean leaflet washout velocity in the leaflets neighborhood region

\begin{tabular}{|c|c|c|c|c|c|c|}
\hline & \multicolumn{3}{|c|}{ Systole-without coronary flow } & \multicolumn{3}{|c|}{ Diastole-without coronary flow } \\
\hline & BPV & $\begin{array}{c}\text { CoreValve } \\
\text { (Medtronic Inc, } \\
\text { Minneapolis, Minn) ViV }\end{array}$ & $\begin{array}{c}\text { Sapien (Edwards } \\
\text { Lifesciences, } \\
\text { Irvine, Calif) ViV }\end{array}$ & BPV & CoreValve ViV & Sapien ViV \\
\hline $\begin{array}{l}\text { Leaflet washout } \\
\text { velocity }(\mathrm{m} / \mathrm{s})\end{array}$ & $0.16 \pm 0.23$ & $0.12 \pm 0.096$ & $0.055 \pm 0.072$ & $0.030 \pm 0.036$ & $0.023 \pm 0.044$ & $0.030 \pm 0.034$ \\
\hline \multirow[t]{3}{*}{ Mean shear stress $(\mathrm{Pa})$} & $0.076 \pm 0.55$ & $0.014 \pm 0.48$ & $0.013 \pm 0.067$ & $0.036 \pm 0.081$ & $0.023 \pm 0.093$ & $0.030 \pm 0.052$ \\
\hline & \multicolumn{3}{|c|}{ Systole-with coronary flow } & \multicolumn{3}{|c|}{ Diastole-with coronary flow } \\
\hline & BPV & CoreValve ViV & Sapien ViV & BPV & CoreValve ViV & Sapien ViV \\
\hline $\begin{array}{l}\text { Leaflet washout } \\
\text { velocity }(\mathrm{m} / \mathrm{s})\end{array}$ & $0.41 \pm 0.30$ & $0.19 \pm 0.14$ & $0.15 \pm 0.10$ & $0.21 \pm 0.13$ & $0.20 \pm 0.18$ & $0.17 \pm 0.090$ \\
\hline Mean shear stress $(\mathrm{Pa})$ & $0.31 \pm 0.53$ & $0.12 \pm 0.38$ & $0.2 \pm 0.63$ & $0.24 \pm 0.71$ & $0.078 \pm 0.43$ & $0.14 \pm 0.28$ \\
\hline
\end{tabular}

the CoreValve ViV is not significant, but the BPV pressure gradient is drastically different because the leaflets are in good condition.

\section{DISCUSSION}

We focus our discussion mainly on how ViV changes aortic sinus hemodynamics and its potential relevance to prosthetic leaflet washout and leaflet opening in the context of understanding the mechanisms for leaflet thrombosis, in addition to highlighting the differences in sinus hemodynamics between the CoreValve and the Sapien ViV.

\section{Valve-in-Valve Hemodynamic Effects}

Examination of detailed sinus flow patterns for the ViV cases yields numerous differences from the isolated bioprosthetic valve configuration. Both $\mathrm{ViV}$ vortices are weaker overall than the isolated bioprosthetic valve configuration vortex, with that of the Sapien ViV being stronger than that of the CoreValve ViV but concentrated more downstream away from the leaflets.

The differing vorticity dynamics between these 2 valve cases are regulated largely by geometric and structural changes. When no TAV is present, the leaflet tip opens into the sinus during early systole. In the presence of a CoreValve, on the other hand, the leaflet extends farther downstream and is restricted from opening completely by the stent frame. The extended length of the TAV leaflet is likely the reason for the downstream location at which the vortex develops, whereas the constant vortex topology is due to the static BPV leaflet location. With the presence of the Sapien in $\mathrm{ViV}$, the leaflets do not extend much beyond those of the BPV and the vortex develops downstream away from the leaflets, which highlights the more significant impact of the stent structure.

The vortex structure also is due to viscous effects and geometry. These effects and their impact on vortex formation and development are explained for general sinus flow by Yap and colleagues. ${ }^{16}$ In summary, a vortex forms within a sinus by (1) some of the free-stream flow entering the sinus or (2) shear force interaction between the sinus fluid and the free-stream flow. In the present study, it appears that the free-stream flow entering the sinus is responsible for the BPV case sinus vortex and the shear force interaction between the sinus fluid and the free-stream flow is responsible for the $\mathrm{ViV}$ sinus vortices. This means that no free-stream flow enters the sinus in the ViV cases, which could be due to the extension of the leaflet in the case of the CoreValve $\mathrm{ViV}$, the restriction of leaflet opening by the stent, by the stent itself, or by a combination of these factors. Regardless of the cause, the absence of free-stream flow in the ViV case sinus leads to a weaker vortex, with flow shear being the sole contributing factor behind vortex formation. In addition, some quiescent fluid near the BPV leaflet is entrained by the vortex, thus increasing overall fluid motion from early to midsystole.

Altered vorticity dynamics due to the implantation of a TAV could have implications from the standpoint of clinical and subclinical leaflet thrombosis. This is mainly due to the low magnitude and downstream location of the TAV sinus vortex. Low magnitude means low blood velocity and therefore low shear stress along the leaflet, a factor that is strongly linked to leaflet thrombosis. Likewise, a downstream vortex that only slightly reaches the base of the sinus leaves a pocket of nearly stagnant fluid for much of the cardiac cycle. This particular finding is in agreement with results from a study by Ducci and colleagues. ${ }^{20}$ Such stagnation zones often are associated with thrombosis, which could explain an increased risk of leaflet thrombosis and elevated gradients for patients with TAV.,10

\section{Valve-in-Valve Hemodynamic Effects in a Coronary Sinus}

Most alterations in hemodynamics brought about by TAV implantation within the BPV occur in both noncoronary and 


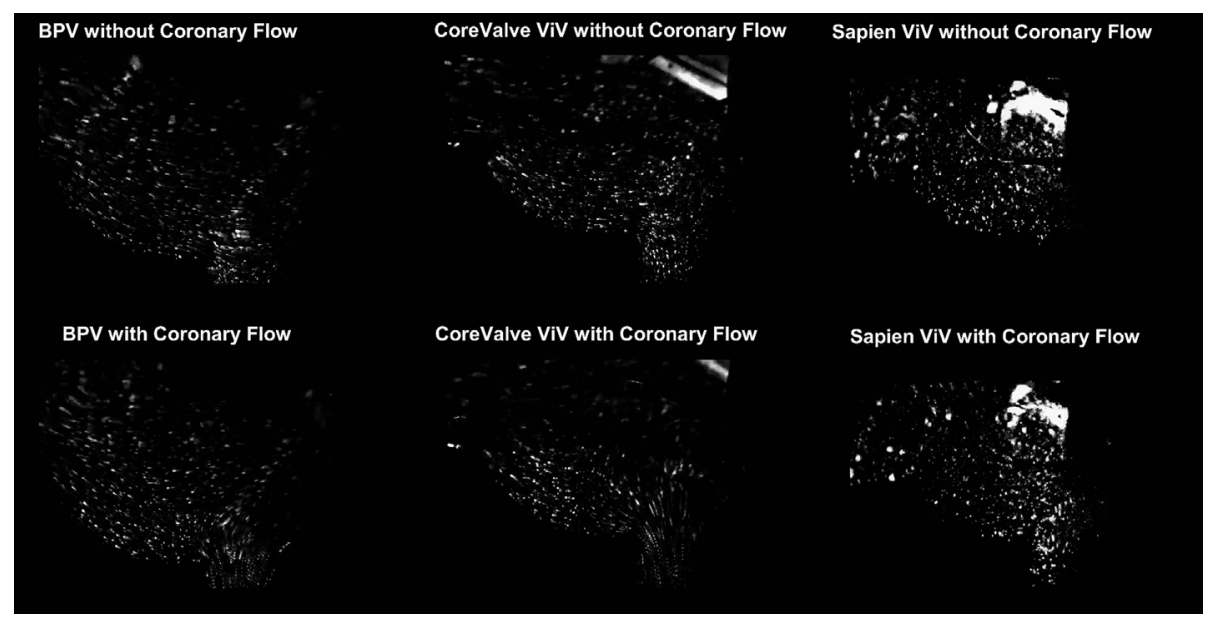

VIDEO 1. High-speed sinus flow visualization for all cases. Particle streak videos along the centerline of the noncoronary sinus on the top row and the coronary sinus on the bottom row. Forward flow is right to left, and the aortic cusp may be seen to open on the right hand side of each video. BPV sinus flow visualization without coronary flow: During systole, sinus vortex forms and is quickly positioned along the middle of the sinus length. The absence of coronary inflow into the ostium both during systole and diastole creates a weak flow pattern adjacent to the leaflets. BPV sinus flow visualization with coronary flow: During systole, sinus vortex forms and is quickly positioned adjacent to the leaflet because of the suction from the coronary ostium. Coronary inflow at the ostium appears helical. Diastolic flow shows sinus flow pattern dominated by the flow into coronary ostium. Consequently, there is more flow adjacent to the leaflets. ViV with CoreValve (Medtronic Inc, Minneapolis, Minn) sinus flow visualization without coronary flow: During systole, sinus vortex forms and is slowly positioned adjacent to the prosthesis leaflet. There is poor sinus circulation adjacent to the leaflet. Absence of coronary inflow into the ostium both during systole and diastole creates a weak circulation adjacent to the leaflets. ViV with CoreValve sinus flow visualization with coronary flow: During systole sinus vortex forms and is quickly positioned adjacent to the prosthesis leaflet. Two vortices exist during forward flow. Coronary inflow at the ostium appears straight. Diastolic flow shows sinus flow pattern dominated by the flow into coronary ostium. Consequently, there is more flow adjacent to the leaflets. ViV with Sapien (Edwards Lifesciences, Irvine, Calif) sinus flow visualization without coronary flow: During systole, sinus vortex forms and is positioned close to the sinotubular junction away from the Sapien leaflet. There is poor sinus circulation adjacent to the leaflet. Absence of coronary inflow into the ostium both during systole and diastole creates a weak circulation adjacent to the leaflets. ViV with Sapien sinus flow visualization with coronary flow: During systole, sinus vortex forms and the flow follows the contour of the sinus wall led by the coronary flow. The vortex is away from the Sapien leaflet. Coronary inflow at the ostia appears straight. Diastolic flow shows sinus flow pattern dominated by the flow into coronary ostium. Consequently, there is more flow adjacent to the leaflets. BPV, Bioprosthetic heart valve; ViV, valve-in-valve. Video available at: http://www.jtcvsonline.org/article/ S0022-5223(17)30563-9/addons.

coronary sinuses. However, some hemodynamic changes are unique to the coronary sinus. Flow into the ostium represents a larger portion of fluid movement in the sinus. Furthermore, this added flow could help alleviate thrombosis risk in the coronary sinus of $\mathrm{ViV}$ patients, a mechanism we elude to explain preferential leaflet thrombosis only in leaflets that are protected from the benefits of coronary flow. ${ }^{5}$

\section{Shear Stress}

Leaflet wall shear stress often is of interest in aortic valve studies because of its correlation to thrombosis and calcification in native and prosthetic leaflets. Although no wall shear stress was calculated in this study, fluid shear stress within the sinus was calculated and represents the scale of magnitude of wall shear stresses at leaflet at different times during the cardiac cycle.

Coronary presence alone alters some sinus shear stress patterns in the isolated bioprosthetic valve configuration mainly the leaflet. When examining this region in both cases, it is apparent that there is higher magnitude shear for the coronary sinus. In addition, shear stress direction is constant for the coronary case, whereas it changes direction for the noncoronary case. Both of these trends have implications for disease because reduced and oscillatory shear stresses are correlated to development of thrombosis or calcification. ${ }^{21}$ Therefore, the noncoronary sinus appears hemodynamically more susceptible to thrombosis or calcification than the coronary sinus, which is supported by clinical findings that show earlier and more frequent signs of calcification on the noncoronary leaflet. ${ }^{22}$

When examining the effects of the $\mathrm{ViV}$ arrangement on shear stress in the sinus, the most noticeable trend is that lower magnitude shear stress occurs in the ViV valve cases. Such a large reduction in shear could lead to accelerated calcification of the prosthesis leaflets and growth of thrombosis leading to leaflet mobility problems. ${ }^{2,5}$

Coronary presence significantly increases sinus shear stresses in the $\mathrm{ViV}$ sinus throughout the cardiac cycle, most notably along the aortic side of the leaflet. This could 
be caused by a low pressure region near the ostium that helps pull the vortex toward the upstream end of the sinus. Lower ostial pressure ("sink hole effect") also produces some shear stress in the base of the sinus from entrainment of otherwise stagnant flow at the base into the ostium from this region.

\section{Study Limitations}

This study was performed in vitro in a rigid aortic chamber using 1 size for each of the TAVs used to fit in the BPV with leaflets within the post, a 26-mm Medtronic CoreValve and a 23-mm Edwards Sapien. Future studies will entail surgical BPVs with leaflets on the outside of the post.

\section{CONCLUSIONS}

This study constitutes a detailed look at aortic sinus hemodynamics as regulated by a $\mathrm{ViV}$ arrangement analyzed with and without the presence of coronary flow. Novel methodology was developed to simulate coronary flow in vitro. Sinus flow patterns were greatly altered with the introduction of a TAV whether CoreValve or Sapien compared with BPV (non-ViV) in a way that peak sinus flow velocity was reduced and a particular lack of sinus flow was evident toward the base in both ViV cases. These trends could help explain the risk of leaflet thrombosis in patients with ViV. The coronary sinus demonstrated higher flow velocities and wall shear stresses than the noncoronary sinus, meaning that this sinus may be less susceptible to thrombus formation.

\section{Conflict of Interest Statement}

Dr Crestanello reports grants from Medtronic, Boston Scientific, and Abbott and work on Medtronic Advisory board. Dr Moore reports employment by St. Jude Medical. All other authors have nothing to disclose with regard to commercial support.

\section{References}

1. Bapat V, Attia R, Redwood S, Hancock J, Wilson K, Young C, et al. Use of transcatheter heart valves for a valve-in-valve implantation in patients with degenerated aortic bioprosthesis: technical considerations and results. J Thorac Cardiovasc Surg. 2012;144:1372-80.

2. Makkar RR, Fontana G, Jilaihawi H, Chakravarty T, Kofoed KF, de Backer O, et al. Possible subclinical leaflet thrombosis in bioprosthetic aortic valves. $N$ Engl J Med. 2015;373:2015-24.
3. De Marchena E, Mesa J, Pomenti S, Marin Y, Kall C, Marincic X, et al. Thrombus formation following transcatheter aortic valve replacement. JACC Cardiovasc Interv. 2015;8:728-39.

4. De Marchena E, Mesa J, Pomenti S, Marin Y, Kall C, Marincic X, et al. Thrombus formation following transcatheter aortic valve replacement. JACC Cardiovasc Interv. 2015;8:1140-1.

5. Hatoum H, Crestanello J, Dasi LP. Possible subclinical leaflet thrombosis in bioprosthetic aortic valves. $N$ Engl J Med. 2016;374:1590-2.

6. Groves EM, Falahatpisheh A, Su JL, Kheradvar A. The effects of positioning of transcatheter aortic valves on fluid dynamics of the aortic root. ASAIO J. 2014;60: 545-52.

7. Dvir D, Webb J, Brecker S, Bleiziffer S, Hildick-Smith D, Colombo A, et al. Transcatheter aortic valve replacement for degenerative bioprosthetic surgical valves: results from the global valve-in-valve registry. Circulation. 2012;126:2335-44.

8. Stanger O, Gisler F, Londono MC. Valve-in-valve: estimating the risk of ostium obstruction in failed Sorin stentless valves. J Thorac Cardiovasc Surg. 2015;149: 644-5.

9. Waksman R, Minha SA. Stroke after aortic valve replacement: the known and unknown. Circulation. 2014;129:2245-7.

10. Smith CR. Transcatheter versus surgical aortic-valve replacement in high-risk patients. N Engl J Med. 2011;364:2187.

11. Fanning JP, Wesley AJ, Platts DG, Walters DL, Eeles EM, Seco M, et al. The silent and apparent neurological injury in transcatheter aortic valve implantation study (SANITY): concept, design and rationale. BMC Cardiovasc Disord. 2014;14:45.

12. Brown ML, Park SJ, Sundt TM, Schaff HV. Early thrombosis risk in patients with biologic valves in the aortic position. J Thorac Cardiovasc Surg. 2012;144:108-11.

13. Tseng EE. When valve-in-valve implantation is not sufficient: bioprosthetic Russian dolls. J Thorac Cardiovasc Surg. 2016;152:624-5.

14. Moore B, Dasi LP. Spatiotemporal complexity of the aortic sinus vortex. Exp Fluids. 2014;55:1770.

15. Moore BL, Dasi LP. Coronary flow impacts aortic leaflet mechanics and aortic sinus hemodynamics. Ann Biomed Eng. 2015;43:2231-41.

16. Yap CH, Saikrishnan N, Tamilselvan G, Yoganathan AP. Experimental measurement of dynamic fluid shear stress on the aortic surface of the aortic valve leaflet. Biomech Model Mechanobiol. 2012;11:171-82.

17. Lichtenstein SV, Cheung A, Ye J, Thompson CR, Carere RG, Pasupati S, et al Transapical transcatheter aortic valve implantation in humans: initial clinical experience. Circulation. 2006;114:591-6.

18. Bapat VN, Attia RQ, Condemi F, Visagan R, Guthrie M, Sunni S, et al Fluoroscopic guide to an ideal implant position for Sapien XT and CoreValve during a valve-in-valve procedure. JACC Cardiovasc Interv. 2013 6:1186-94.

19. Azadani AN, Reardon M, Simonato M, Aldea G, Nickenig G, Kornowski R, et al. Effect of transcatheter aortic valve size and position on valve-in-valve hemodynamics: an in-vitro study. J Thorac Cardiovasc Surg. 2017;153: 1303-15.e1.

20. Ducci A, Tzamtzis S, Mullen MJ, Burriesci G. Hemodynamics in the Valsalva sinuses after transcatheter aortic valve implantation (TAVI). J Heart Valve Dis. 2013;22:688-96

21. Chandra S, Rajamannan NM, Sucosky P. Computational assessment of bicuspid aortic valve wall-shear stress: implications for calcific aortic valve disease. Biomechan Model Mechanobiol. 2012;11:1085096.

22. Freeman RV, Otto CM. Spectrum of calcific aortic valve diseasepathogenesis, disease progression, and treatment strategies. Circulation. $2005 ; 111: 3316-26$

Key Words: aortic sinus, calcification, thrombosis, transcatheter aortic valve implantation 


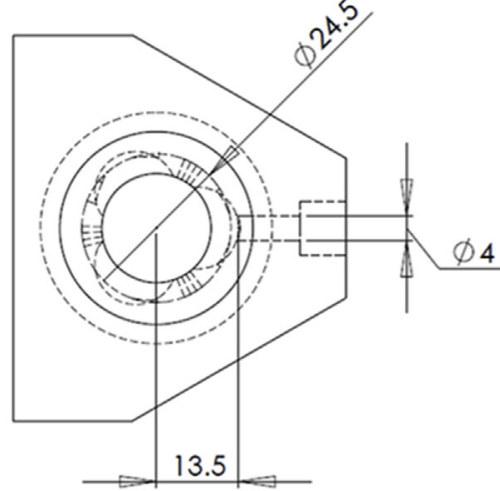

A

B

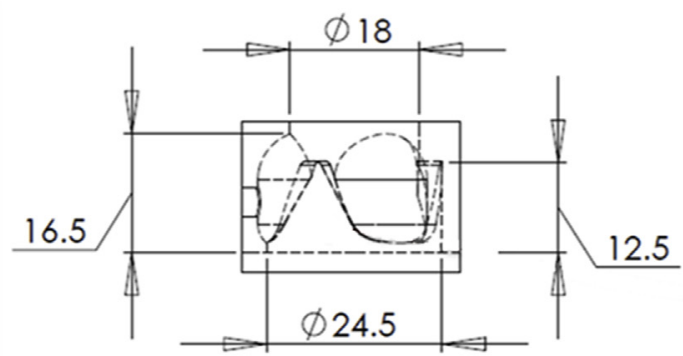

c

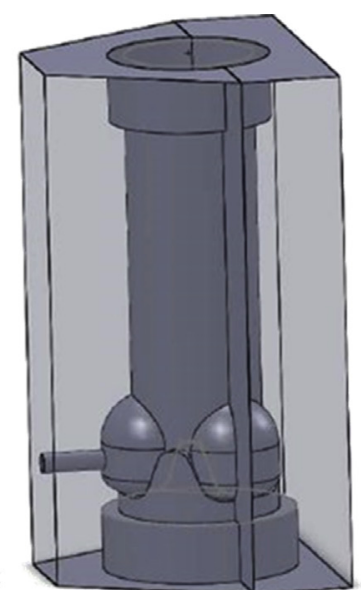

FIGURE E1. Detailed sinus chamber dimensions are shown from the (A) top view, (B) side view, and (C) 3-dimensional model of the aortic valve chamber.

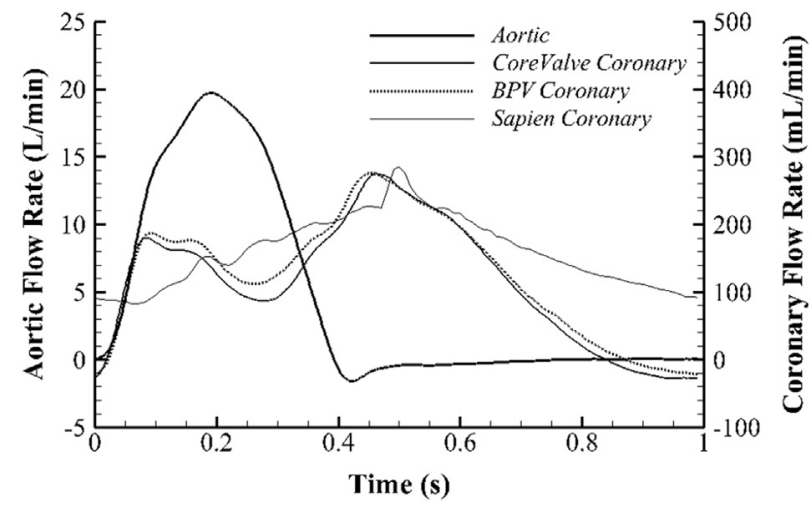

FIGURE E2. Left coronary waveforms for isolated BPV and ViV configurations along with native aortic flow waveform. The coronary circuit was designed to provide physiologic coronary flows throughout the cardiac cycle through the use of a pneumatically controlled Starling resistor. Namely, this resistor was collapsed or expanded during specific intervals to match the changes in coronary flow during myocardial isovolumetric contraction and relaxation, respectively. A small compliance chamber was used to modulate the compressive forces. ${ }^{15} \mathrm{BPV}$, Bioprosthetic heart valve.

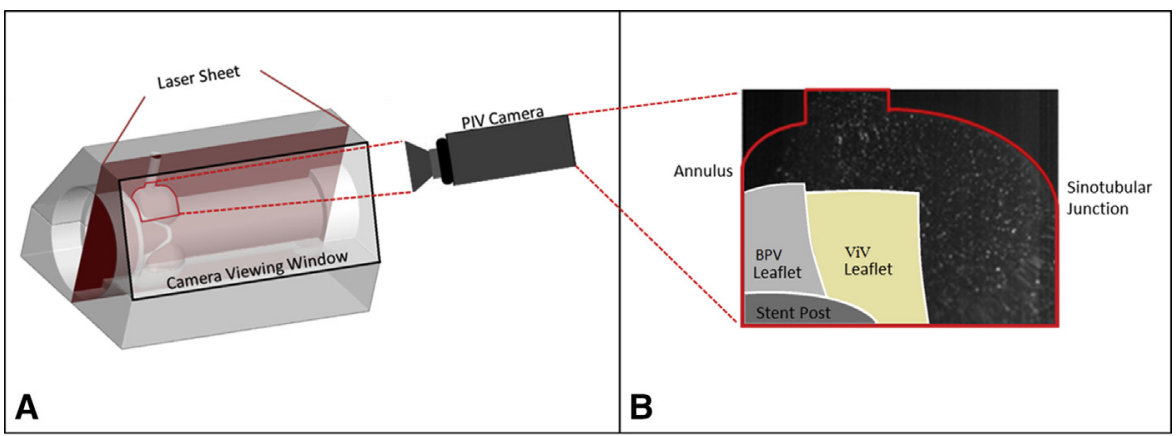

FIGURE E3. Image of (A) transparent acrylic valve chamber with depiction of laser sheet and camera viewing plane. The coronary sinus that was studied is outlined in red. B, Raw camera image of sinus, with annotated valve leaflets, used for particle image velocimetry calculations. PIV, Particle image velocimetry; $B P V$, bioprosthetic heart valve; $V i V$, valve-in-valve. 